\title{
Dénoncer les maltraitances gynécologiques en URSS : critique ordinaire des patientes et critique féministe (1979-1989)
}

\author{
par \\ Mona Claro
}

Si les concepts de « violences obstétricales » et de « maltraitances gynécologiques » ont émergé dans les années 2000-2010, les critiques qu'ils cristallisent ne sont pas nouvelles (Vuille 2016) ${ }^{1}$. Des dénonciations de souffrances physiques et psychologiques évitables, d'actes médicaux injustement imposés ou refusés, et plus largement de rapports de pouvoir patiente-médecin trop asymétriques, dans les domaines de la contraception, de l'avortement et de l'accouchement notamment, se faisaient déjà entendre dans les décennies précédentes. Celles qui proviennent des féminismes de la «deuxième vague» des pays occidentaux font l'objet d'une littérature scientifique déjà fournie (Löwy 2005 ; Morgen 2002 ; Ruault 2017). Par contraste, les travaux sur ces enjeux qui abordent l'URSS insistent sur l'absence de telles mobilisations féministes ou de patientes, dans ce contexte autoritaire (Michaels 2014 : 155, 139 ; Riska 2001 : 117-119).

Or des sources existent, qui n'ont pas encore été analysées en profondeur sous cet angle. Au début des années 1980, des dissidentes féministes ont développé une critique de la médecine soviétique à partir de leurs expériences de patientes en gynécologie, dans une série de publications clandestines («samizdat»). Comme l'a noté Sue Bridger (1990), à l'époque, la façon dont elles rendaient compte de leurs avortements et de leurs accouchements tranchait radicalement avec les silences et les discours enchantés des publications officielles, mais avec la perestroïka, dès 1986, des témoignages et des indignations très similaires sont apparus dans la presse féminine soviétique officielle, notamment dans de nombreuses lettres de lectrices publiées. Les dissidentes, puis les lectrices de la "glasnost», ont dénoncé un personnel soignant indifférent à leurs douleurs physiques, «froid » et débordé, voire « cruel», et ont parlé des soins gynécologiques en termes «tortures»- bref, elles ont mobilisé le même champ lexical de la maltraitance. Cet article s'intéresse à ces critiques ordinaires et dissidentes, à travers deux ensembles de sources

\footnotetext{
${ }^{1}$ Je remercie Anna Sidorevich et Lena Smirnova pour l'aide précieuse qu'elles m'ont apportée dans la rédaction de cet article, en termes d'accès aux sources et à la littérature scientifique.
} 
russophones : les samizdats féministes Femme et Russie et Maria (8 numéros parus en 19791984) ${ }^{2}$, d'une part, le magazine féminin Rabotnitsa ou La Travailleuse (5 numéros parus en 19861989) ${ }^{3}$, d'autre part.

Des travaux sur l'histoire des féminismes occidentaux de la deuxième vague se sont penchés sur « les appropriations ordinaires des idées féministes » (Jacquemart et Albenga 2015), et ont identifié une «diffusion par capillarité des idées féministes » jusque chez des femmes qui «ne s'auto-désignent pas ainsi » et qui n’ont « que très peu connaissance de la réalité du mouvement » (Achin et Naudier 200 : 139). Bibia Pavard (2017) en particulier s'est intéressée à la diffusion dans la presse féminine d'un «féminisme populaire ». Le cas de la presse féminine soviétique de la perestroïka permet de réexaminer cette question à nouveaux frais. En effet, en URSS, un régime autoritaire a longtemps participé à rendre le militantisme féministe non seulement improbable, mais aussi inaudible. Avec la "glasnost », l'assouplissement de la censure a permis l'expression dans Rabotnitsa de témoignages et de revendications féminines faisant écho à celles formulées quelques années auparavant par des féministes dissidentes, alors même que ces écrits militants étaient largement méconnus des journalistes comme des lectrices. Rabotnitsa ne faisait aucune référence aux samizdats, ni d'ailleurs au féminisme en général. Ce cas invite à se poser non pas la question des réappropriations du féminisme par les femmes ordinaires, mais plutôt la question de comment les mobilisations féministes sont nourries par des indignations féminines ordinaires qui les précèdent et les dépassent. À la suite de Lucile Quéré (2019), j’emprunte à Fabrizio Cantelli (2015) le concept de «critique ordinaire du patient» pour analyser des indignations qui ne prennent pas nécessairement la forme de recours formels ou de mobilisations collectives. Il s'agit de « prendre au sérieux toute manifestation du sens critique des patients : celle qui s'exprime à bas bruit comme celle qui s'exprime à haute voix », « celle qui se partage entre proches [...] comme celle qui va vers les médias » (ibid. : 108). Cet article se propose d'analyser les conditions d'émergence de critiques ordinaires et féministes de la gynécologie dans la période soviétique tardive. J'examinerai leurs points de convergence, leurs contenus et leurs limites, en les mettant en perspective avec des comparaisons internationales.

\footnotetext{
${ }^{2}$ Cet article s'appuie sur la consultation exhaustive des quatre premiers numéros de Femme et Russie et du premier numéro de Maria. En raison de difficultés d'accès aux sources, le $5^{\mathrm{e}}$ numéro de Femme et Russie et les trois numéros suivants de Maria n'ont pu être consultés que partiellement. Ces sources étaient accessibles en langue originale (russe) sauf, pour Femmes et Russie, les numéros 2 à 4 (traduction en français) et les extraits du $5^{\mathrm{e}}$ (traduction en anglais).

${ }^{3} \mathrm{~N}^{\circ} 10$ de $1986 ; \mathrm{n}^{\circ} 7$ de $1987 ; \mathrm{n}^{\circ} 4$ de $1988 ; \mathrm{n}^{\circ} 2$ et 4 de 1989.
} 


\section{Un silence officiel assourdissant}

Les samizdats féministes ont émergé à la fin de l'ère Brejnev. À l'époque, la santé sexuelle et reproductive des femmes soviétiques était gouvernée selon le mode du dénuement, de l'inertie et du secret. Officiellement, les soins étaient gratuits, et des médecins «de secteur» étaient assigné $\cdot e \cdot s$ automatiquement. Toutefois, dans les faits, il était relativement courant de contourner les règles bureaucratiques dans l'espoir de s'assurer des soins de meilleure qualité, en choisissant «sa/son » médecin « par interconnaissance », et en lui versant des pots-de-vin («blat») (RivkinFish 2005). Dans l'économie planifiée, les dépenses industrielles et militaires étaient prioritaires par rapport à celles de santé. Entre 1965 et 1985, la part des dépenses de santé dans le PNB était en baisse et proche de 5\%, contre 10\% dans la plupart des pays occidentaux (Riska 2001 : 93). Les établissements médicaux étaient souvent «surchargés », manquaient d'équipements médicaux et de produits pharmaceutiques, n'étaient «pas correctement entretenus», voire insalubres (Davis 1998). Par ailleurs, les médecins étaient des fonctionnaires sans autonomie professionnelle, aux salaires modestes, et essentiellement des femmes, surtout en bas des hiérarchies hospitalières elles représentaient environ $70 \%$ des médecins, et en particulier plus de $90 \%$ des gynécologues (Riska 2001 : 81). Deux indicateurs de santé publique, jugés alarmants et susceptibles de discréditer le régime, étaient tenus secrets: les taux d'avortements et de mortalité infantile étaient inaccessibles non seulement au grand public, mais aussi aux scientifiques et aux autorités compétentes, à l'exception de quelques hauts fonctionnaires (Blum 2018). C'est seulement pendant la perestroïka que le pays a découvert, en 1988, qu'il avait le taux d'IVG le plus élevé au monde, et que sa mortalité infantile, repartie à la hausse vers 1970, était élevée, comparée à celle des pays de l'Ouest. La «glasnost » a également permis l'expression d'une indignation inédite au sujet de la mortalité maternelle : en 1990, le taux soviétique était proche de 50 pour 100 000, contre 10 environ en Union Européenne (John Snow Inc. 2012).

L'avortement sur demande, légalisé en 1920, interdit sous Staline, puis de nouveau autorisé en 1955, était considéré par les autorités comme un mal nécessaire : le régime mettait en scène ses efforts pour permettre aux femmes d'élever de nombreux enfants tout en travaillant à temps plein, mais reconnaissait l'inéluctabilité du contrôle des naissances, et cherchait à éviter les risques sanitaires des avortements clandestins. Sur le papier, le ministère de la Santé reconnaissait que la contraception devait être plus répandue et plus efficace, pour faire diminuer le recours à l'IVG, 
mais dans les faits, les efforts dans ce sens étaient limités. Dans la presse grand public de la fin des années 1970, les couples mariés et déjà parents pouvaient se voir conseiller d'utiliser la méthode du calendrier, le préservatif ou le stérilet. Toutefois, toujours en raison des priorités de l'économie planifiée, des problèmes de pénurie et de qualité des contraceptifs se posaient. De plus, la pilule, considérée par le ministère comme dangereuse, se voyait rarement prescrite. Dans ce domaine, les statistiques n'étaient pas secrètes, mais tout simplement quasi inexistantes : avant 1988, les prévalences des différences méthodes contraceptives ne faisaient l'objet d'aucune collecte statistique de grande ampleur (Claro 2018: 79-117). Selon des estimations calculées ultérieurement, en 1979, seules 0,5\% des femmes soviétiques de 15-49 ans en union prenaient la pilule, et $1,5 \%$ avaient un stérilet; à l'aube de la perestroïka, la prévalence de la pilule restait inférieure à 1\%, tandis que celle du stérilet s'approchait de 10\% (Avdeev et Troitskaia 1991). Les samizdats féministes ont donc émergé dans un contexte où le gouvernement soviétique, après des années d'immobilisme, commençait tout juste à investir dans la contraception médicalisée, en orientant ses efforts modérés non pas vers le choix des usagères, mais vers une seule méthode, réservée aux femmes déjà mères : le stérilet, moins onéreux que la pilule. Il faudra attendre la perestroïka pour lire dans la presse, d'une part, que les femmes soviétiques avortaient en moyenne 3 ou 4 fois au cours de leur vie ${ }^{4}$, d'autre part, que 80 à $95 \%$ des avortements étaient réalisés « sans anesthésie efficace », voire « sans aucune anesthésie $»^{5}$. Concernant les méthodes employées, là encore, l'immobilisme et le silence étaient de mise : dans les pays de l'Ouest qui avaient légalisé l'IVG, l'aspiration avait rapidement supplanté le curetage ; en URSS, les femmes n'entendront parler publiquement de cette méthode moins risquée et moins douloureuse qu'en 1988, lorsque le ministère se décidera à la promouvoir (Claro 2018 : 124-126).

Dans le domaine de l'obstétrique, comme l'a montré Paula Michaels (2014), dans les années 1960-80, l'URSS est restée à l'écart de trois grandes évolutions qui ont touché les façons d'accoucher en Occident. Premièrement, à partir des années 1960, la diffusion de méthodes de préparation à la naissance et d'accouchement émanant ou inspirées de médecins comme Grantly Dick Read, Ilya Velvovski, Ferdinand Lamaze, Frédérick Leboyer, ou Michel Odent, qui furent accueillies positivement par une partie des féministes. Deuxièmement, en lien direct avec cette première évolution, un mouvement d'inclusion de personnes proches accompagnantes dans les

\footnotetext{
${ }^{4}$ Zdorov'e, 1988, n 3

${ }^{5}$ Zdorov'e, $1988, \mathrm{n}^{\circ} 1$.
} 
accouchements, essentiellement le père. Troisièmement, à partir des années 1980, la diffusion de l'anesthésie péridurale. La description par Yvonne Knibielher du modèle d'accouchement qui prévalait en France dans les années 1950-60 se superpose parfaitement avec celle faite par Michele Rivkin-Fish $(2005: 25,40)$ du protocole resté standard jusque dans les années 1990, en URSS :

Une femme qui entre en clinique quitte les siens, elle se met aux ordres des médecins - pour ne pas dire à leur merci. [...]. Ils traitaient les mères, les parents, du haut de leur science, sans égards, parfois avec brutalité ; et les sages-femmes, réduites au rang d'auxiliaires, suivaient cet exemple. Les accouchements se faisaient à la chaîne [...]. Des rites inspirés de l'hygiène pasteurienne (lavement, rasage du pubis, immobilisation à plat dos sur une table étroite) humiliaient la patiente, la dépossédaient de son corps. On la séparait de son bébé, qui lui était apporté seulement toutes les trois heures pour une tétée limitée à un quart d'heure, et donnée obligatoirement en position assise. Par crainte des microbes, on écartait le père et les proches. (Knibiehler $2010: 40$ )

Il est paradoxal de constater que les douleurs lors des accouchements ne faisaient l'objet de presque aucune prise en charge, ni pharmacologique, ni de l'ordre de l'accompagnement, alors que l'URSS était considérée ailleurs dans le monde comme un pays pionnier de « l'accouchement sans douleur ». C'est le psychologue soviétique Ilya Velvovski qui a inventé en 1948 la méthode "psychoprophylactique », connue plus tard en Occident en tant que «méthode Lamaze» (Michaels 2014 : 74). En s'inspirant des travaux de Pavlov sur le conditionnement, Velvovski affirmait que les parturientes pouvaient apprendre à empêcher leurs douleurs en étant soigneusement éduquées au processus de l'accouchement, et grâce à des techniques de respiration et de relaxation. À l'époque, aucune technique pharmacologique contre les douleurs de l'accouchement ne faisait un tant soit peu l'unanimité : à travers le monde, les médecins pouvaient utiliser divers produits, comme le chloroforme ou l'opium, qui n'étaient pas sans risques (Vuille 2017). La méthode de Velvovski contournait ce problème, et fut décrétée méthode d'accouchement officielle en URSS, via une directive de 1951. Selon Paula Michaels, cette méthode a triomphé, malgré les réticences de la profession médicale, notamment car elle était compatible avec les pénuries de produits anesthésiants. Velvovski ne mesurait pas l'efficacité de sa méthode via une auto-déclaration par la parturiente de la douleur ressentie, mais en attribuant lui-même une note basée sur ses observations (Hrešanová et Michaels 2018 : 50). Le seul point qui faisait consensus, chez les médecins soviétiques «pour » ou « contre » la méthode, était qu'elle avait l'avantage de discipliner les parturientes et de les faire taire (Hrešanová et Michaels 2018 : 62) : elles étaient entraînées à ne pas « crier » et à ne pas « se contorsionner », et « même si elles ressentaient toujours 
des douleurs », « elles étaient plus faciles à gérer » (Michaels $2014: 80-82)^{6}$. Cette technique est restée la méthode officielle d'accouchement jusqu'à la chute de l'URSS : le ministère «prétendait que [la préparation prophylactique à la naissance] était presque universelle ", mais l'enquête de Michaels « suggère qu'une telle préparation n'existait que sur le papier » (Hrešanová et Michaels 2018: 63). Pour conclure, à la fin de la période soviétique, en matière de contraception, d'avortement et d'accouchement, c'était le manque de moyens, l'immobilisme et l'opacité qui régnaient.

\section{Du témoignage à l'indignation}

C'est donc un silence assourdissant que sont venues briser les dissidentes féministes, dès 1979 - dans leurs écrits clandestins restés confidentiels - puis, à partir de 1986, le magazine féminin Rabotnitsa - touchant des millions de lectrices, à l'heure de la « glasnost ».

\section{Les samizdats féministes}

Les quatre fondatrices des «samizdats » féministes, enfants sous Staline et jeunes adultes pendant le Dégel, se sont d'abord engagées aux côtés d'hommes dans la dissidence et dans la «seconde culture» artistique (clandestine) de Saint-Pétersbourg, avant de se mobiliser entre femmes et pour la cause des femmes. Elles avaient fait des études supérieures, surtout dans le domaine des lettres et sciences humaines, et selon un scénario typique dans leur milieu «non conformiste ", n'ayant pas pu ou pas voulu faire carrière dans des professions intellectuelles exigeant une loyauté vis-à-vis du régime, s'étaient rabattues sur des emplois non qualifiés (Sidorevich 2017 ; Tchouikina 1996). Elles étaient toutes mariées ou divorcées, et presque toutes mères. Parmi elles, c'est Tatiana Mamonova qui a lancé l'idée d'un samizdat féministe dès 1976, après avoir renoncé à faire avancer la cause des femmes depuis l'intérieur des institutions officielles - sa candidature au Comité des femmes soviétiques avait été refusée. À la fin des années 1970, elle a eu accès à des écrits féministes occidentaux circulant clandestinement en URSS, comme des livres de Robin Morgan ou Kate Millett, ou encore le journal Des femmes en mouvements hebdo, qui ont notamment été apportés dans le pays, à la base, par des militantes du courant essentialiste du MLF français («Psych et Po ») (Harting 2013 ; Ruthchild 2017). Les quatre cofondatrices des

6 Toutes les traductions de citations issues de la littérature scientifique ou des sources primaires originellement en anglais ou en russe sont de l'auteure. 
samizdats se sont rejointes pour dénoncer leur position subordonnée dans le mouvement dissident, où les femmes étaient moins publiées que les hommes, souvent reléguées aux rôles d'épouses de prisonniers politiques, d'hôtesses de réunions dans les cuisines, et de dactylos (Ruthchild 2017 ; Tchouikina 1996). Toutefois, après la sortie du premier numéro de Femmes et Russie (ci-après $F \& R$ ) en 1979, dont l'introduction « faisait à la fois l'éloge de Lénine et de Jésus » (Talaver 2017 : 41), le collectif s'est rapidement divisé, en rejouant le clivage qui traversait l'ensemble de la dissidence soviétique. D’un côté, Tatiana Mamonova, selon une posture proche de celle de Andreï Sakharov, était athée et internationaliste, et saluait certains acquis de la Révolution d'Octobre. De l'autre, Youlia Voznesenskaya, Natalia Malakhovskaya et Tatiana Goritcheva, qui s'opposaient radicalement au marxisme, et qui prônaient un féminisme « russe » et chrétien orthodoxe, ont fait sécession juste après la publication du premier numéro, en créant un nouveau samizdat féministe, Maria (en référence à la Vierge Marie), bientôt soutenu par Alexandre Soljenitsyne (Ruthchild 2017).

Les deux courants ont connu une même répression politique, quelque peu atténuée par une campagne de soutien internationale : dès 1980, les quatre cofondatrices, expulsées du pays et déchues de leur nationalité, ont dû poursuivre leur activité de publication depuis l'Ouest. En URSS puis en exil, elles collectaient des textes venant d'un cercle plus large d'auteures (ne se connaissant pas nécessairement mutuellement), de Saint-Pétersbourg mais aussi d'autres villes russes, voire d'autres républiques soviétiques. Entre 1979 et 1984, ce sont finalement cinq numéros de $F \& R$ et trois numéros de Maria qui ont été diffusés de façon extrêmement restreinte en URSS, à raison de 10-12 exemplaires par numéro, passant clandestinement de mains en mains dans les cercles contestataires, et finissant en partie confisqués par les autorités - même par rapport aux autres samizdats, ces tirages étaient très restreints (Sidorevich 2017 : 82). La question de la santé sexuelle et reproductive occupait une place plus ou moins centrale dans les deux revues clandestines ${ }^{7}$, parmi une grande diversité de thèmes, comme la conciliation travail-maternité, le lesbianisme, l'alcoolisme masculin, l'opposition à la guerre en Afghanistan, la renaissance religieuse, ou encore la poésie. Selon Anna Sidorevich (2017 : 38), c'est précisément le récit d'accouchement de Mamonova (publié dans le $1^{\mathrm{e}}$ numéro de $F \& R$ ) qui est considéré par deux autres des cofondatrices comme leur texte fondateur et l'élément déclencheur de leur participation.

\footnotetext{
${ }^{7}$ Cette question est par exemple abordée dans trois textes sur 12 dans le $1^{\text {er }}$ numéro, et dans un ou deux textes sur 21 dans chacun des trois numéros suivant de $F \& R$.
} 


\section{Les lettres de plaintes à la presse féminine}

Quelques années après ces répressions, la perestroïka commençait, et avec elle l'assouplissement de la censure, censé encourager une critique citoyenne constructive. En rendant publiques les statistiques de l'avortement et de la mortalité infantile, les autorités ont ouvert la voie à une critique du système de santé. Le premier magazine mensuel féminin du pays, Rabotnitsafort d'un tirage de 23 millions d'exemplaires en 1990 (Talaver 2017) - a été particulièrement enclin à donner la parole à son lectorat sur ces thèmes et à relayer ses critiques, en sommant les autorités d'y répondre publiquement. Une même séquence s'est répétée deux fois, d'abord sur l'avortement (1986-1987) puis sur l'accouchement (1988-1989). Après la publication d'une première longue lettre de plainte, la rédaction en reçoit des centaines voire des milliers d'autres ${ }^{8}$, dont elle publie des extraits. Ensuite, elle anime dans ses locaux une table ronde de hauts responsables du ministère de la Santé (presque uniquement des hommes), de médecins dirigeant des services ou des établissements, et de démographes (femmes et hommes presque à parité), qui doivent répondre aux courriers du lectorat. Enfin, la retranscription de la table ronde est publiée, entrecoupée de nouveaux extraits de lettres. Les courriers publiés venaient des quatre coins de l'URSS, mais surtout de villes russes, étaient presque toujours signés individuellement, et par des femmes écrivant en tant que patientes - à l'exception, notamment, de la longue lettre d'un homme en ouverture de la séquence sur l'avortement, et d'une lettre collective de soignant·e's sur l'accouchement. Elles ne précisaient presque jamais leur profession, et n'indiquaient jamais si elles étaient membres d'organisations communistes.

En soi, le traitement par la presse de lettres de plainte n'était pas une innovation de la perestroïka. L'institutionnalisation de cette pratique de plainte remontait aux années 1930 : les Soviétiques ordinaires étaient encouragé $\cdot$ 's à envoyer ce qu'on appelait des "signaux » aux autorités, directement ou via la presse, pour les alerter sur les dysfonctionnements du régime. La dénonciation d'une situation pouvait rimer avec de la délation à l'encontre d'individus précis (Nérard 1999). Les lettres de plaintes relevaient d'un genre codifié, instrumentalisé par un régime autoritaire, mais potentiellement «subversif» selon Juliane Fürst (2006). Selon la typologie proposée par Sheila Fitzpatrick (1996), dans les années 1930, on trouvait, d'un côté, les lettres « de citoyens », majoritairement écrites par des hommes, qui donnaient leur avis sur des

\footnotetext{
${ }^{8} 1250$ sur l'avortement, plus de 2500 sur l'accouchement.
} 
dysfonctionnements sans parler de leur situation personnelle, en se présentant comme agissant « dans l'intérêt général ». De l'autre, les lettres « de supplication » étaient le genre le plus souvent investi par les femmes ; celles-ci avaient tendance à écrire « en tant que mères », à raconter leurs difficultés personnelles, et à demander une aide concrète. Il semblerait qu'on puisse retrouver cette pratique de la lettre « de supplication » au cœur de l'activité du magazine Rabotnitsa pendant l'ère Brejnev, étudiée par Alexandra Talaver (2017). Vers 1980, la rédaction de Rabotnitsa recevait environ 500 à 700 lettres par jour, et 15 de ses 35 membres avaient pour mission de les traiter (ibid. : 21, 29). La publication de courriers de lectrices pouvait occuper 2-3 pages par numéro, dominées par un style «sec, parfois bureaucratique », et laissant peu de place aux «émotions » (ibid. : 43). Les lectrices publiées écrivaient essentiellement en tant que travailleuses ou mèrestravailleuses, pour se plaindre de leurs conditions de travail ou de conciliation travail-famille (congés maternité, crèches d'entreprises, etc.). La rédaction relatait ses interventions réussies dans des rubriques comme «Les coupables sont punis » ou « Le ministre a répondu » (ibid. : 31-32). Selon Talaver, le magazine fonctionnait comme un «médiateur entre l'État et les femmes ordinaires », une « plateforme du féminisme d'État » soviétique (ibid. : 19, 34).

La prise en charge par Rabotnitsa, pendant la perestroïka, de plaintes du lectorat au sujet des soins gynécologiques se situait donc dans la continuité de ce "féminisme d'État», mais elle comportait aussi des aspects inédits. Premièrement, les plaintes concernaient des sujets presque jamais abordés auparavant ${ }^{9}$, et prenaient souvent la forme de récits à la première personne, laissant une grande place aux émotions. Deuxièmement, rares étaient les lettres publiées qui orientaient leurs accusations vers des établissements de santé précisément désignés. Les lectrices avaient tendance à demander non pas des interventions ciblées, mais des transformations plus globales, en s'adressant à «notre ministère », à «notre État, notre santé publique ${ }^{10}$. Les genres de la lettre « de supplication » et de la lettre « de citoyen » (Fitzpatrick 1996) semblaient avoir fusionné. Ainsi, avant 1986, le traitement des « signaux » tendait à être individualisant et dépolitisant, à préserver l'ordre établi. Par contraste, pendant la «glasnost », la rédaction s'est appuyée sur les lettres de lectrices pour politiser des souffrances auparavant hors-champ, et pour constituer en problèmes

\footnotetext{
${ }^{9}$ Il semblerait que la seule exception soit un article répondant à une plainte de lectrice au sujet des arrêts de travail suite à un avortement : elle demandait le droit de ne pas dévoiler le motif d'absence à l'employeur (Rabotnitsa, 1977, $\mathrm{n}^{\circ}$ 9).

${ }^{10}$ Rabotnitsa, $1989, \mathrm{n}^{\circ} 2$.
} 
publics l'accès à la contraception, ainsi que la qualité des soins lors des avortements et des accouchements.

3. « On est toutes passées par là »

Dans les samizdats comme dans Rabotnitsa, les auteures dissidentes, les auteures de lettres et les journalistes (là encore, presque toutes des femmes) soulignent à quel point leur prise de parole sur la gynécologie brise un tabou. Le sujet était considéré comme « de mauvais goût », « intime », « honteux ». Dans Rabotnitsa, une lettre s'exclame : «pourquoi on se tait ? [...] Nous vous remercions d'avoir enfin levé ce voile $[\ldots] ! »^{11}$. Du côté de la dissidence féministe comme du côté de la presse féminine, cette prise de parole publique semble avoir été précédée et préparée par des conversations ordinaires entre femmes. Un article de samizdat s'ouvre sur le récit d'un échange entendu entre plusieurs employées de vestiaire et patientes d'un hôpital :

« Pour les femmes, c'est la vie entière qui est une torture ! Tantôt accoucher, tantôt avorter [...]». [...] «- Justement, voilà ! », a ajouté une autre malade, "si les hommes devaient endurer ce que nous endurons, il y a longtemps qu'ils se seraient tous pendus !». Dès le premier mot, ces femmes qui ne se connaissaient pas, qui étaient de générations différentes, et appartenaient à des milieux sociaux différents, sont tombées d'accord ». [...] Ces propos n'ont rien d'exceptionnel. Il suffit de tendre l'oreille, ce sont des paroles courantes. (Collectif $1980 b: 71)$.

De même, plusieurs lettres à Rabotnitsa font référence à des discussions préalables entre femmes autour de la gynécologie, entre collègues notamment - une lettre est d'ailleurs co-signée par 23 ouvrières d'une même usine. Ces sources suggèrent que dans les années précédant la " glasnost », les plaintes au sujet des maltraitances gynécologiques n'étaient pas complètement indicibles, mais plutôt confinées dans des conversations féminines à bas bruit.

Les rédactions des samizdats et de Rabotnitsa s'appuient en partie sur cette pratique sociale préexistante, l'amplifient et la transforment. Elles ne se contentent pas de publier des témoignages, elles les sollicitent aussi activement. De façon révélatrice, le premier samizdat se conclut par cet appel : « Chères sœurs ! [...] Envoyez-nous vos œuvres et vos récits, à propos de vos sœurs, de vos mères, de vos amies » (Collectif 1980a : 123-124). Selon Alexandra Talaver (2017: 65), les rédactrices des samizdats «ont adopté une stratégie de témoignages personnels » proches des « pratiques des groupes de conscience » typiques des féministes occidentales de la deuxième vague.

${ }^{11}$ Rabotnitsa, $1989, \mathrm{n}^{\circ} 2$. 
Ce répertoire d'action a été étudié par Marion Charpenel (2016:23) qui note qu'en « confrontant leurs expériences, les participantes aux groupes peuvent donner à leurs vécus privés une dimension structurelle, les inscrire dans un système. Le collectif fait prendre conscience du caractère politique d'expériences vécues isolément et perçues comme des problèmes individuels ». Le slogan «le privé est politique » est emblématique de ce répertoire. L'un des samizdats féministes met en avant une formule qui y fait écho : «Tant que les ordures n'auront pas été enlevées, on ne nettoiera pas l'izba » (Collectif 1980b : 74), détournement de l'expression russe traduisible par «il faut laver son linge sale en famille». Cette importance donnée à la montée en généralité à partir d'une agrégation de témoignages se retrouve dans Rabotnitsa. On y discerne une démarche qui rappelle celle du magazine féminin français Marie Claire dans les années 1960, analysée par Bibia Pavard (2009) comme le recueil d'un «cahier de doléance de la contraception ». Ainsi, les samizdats et Rabotnitsa ne fournissent pas seulement des instantanés de plaintes féminines ordinaires, ils rendent possible un déplacement par rapport au fatalisme de ces plaintes. Comme l'écrit une lectrice de la «glasnost » : « [après mon accouchement], des femmes plus expérimentées m'ont dit: "ne t'en fais pas, on est toutes passées par là". Mais justement, c'est bien ça qui fait encore plus peur $»^{12}$.

L'indignation nait souvent de la comparaison. Les auteures de textes dissidents ou de lettres à Rabotnitsa construisent une large part de leurs plaintes ou revendications en comparant leurs expériences personnelles de l'accouchement ou de l'avortement, soit entre elles, soit avec les expériences d'autres patientes. Étant donné le silence institutionnalisé qui régnait dans ce domaine avant la perestroïka, le principal, voire le seul moyen de déconstruire l'évidence d'une pratique médicale était de découvrir d'autres possibles par soi-même ou dans les conversations féminines ordinaires. D'après les deux corpus, il était banal de constater que les modalités des soins pouvaient varier fortement d'un espace géographique à un autre (même au sein des seules frontières du bloc de l'Est), et surtout en fonction du recours ou non à des arrangements et des paiements informels («blat $»)$. Parce que certaines femmes avaient entendu parler de, ou avaient vécu, des avortements bien anesthésiés (typiquement, grâce à des dessous-de-table), ou encore des accouchements accompagnés par un personnel disponible et bienveillant (par exemple, en $\mathrm{RDA}^{13}$ ), elles pouvaient comparer, s'indigner, et exiger des changements. Finalement, dans les sources étudiées ici, la

${ }^{12}$ Rabotnitsa, 1988, n¹1.

${ }^{13}$ Rabotnitsa, $1989, \mathrm{n}^{\circ} 2$. 
comparaison joue de deux manières. Tout d'abord, lorsque les femmes comparent leurs expériences de la gynécologie, soit via des discussions, soit plus directement, via le partage de vastes chambres collectives avec une dizaine d'autres patientes, lors des avortements et des accouchements, elles comprennent que leurs souffrances ne sont pas individuelles mais structurelles. Ensuite, d'autres comparaisons révèlent que certaines expériences de soin sont plus enviables que d'autres, et peuvent être érigées en modèle de ce pour quoi il faut lutter. En outre, quand il n'y a ni discours publics ni comparaisons possibles, il est très difficile de s'indigner et de revendiquer: comme on le verra plus loin, certains aspects des soins gynécologiques sont en quelque sorte condamnés à rester impensés.

\section{Des critiques et des impensés}

1. Le destin de l'accouchement et la question de l'avortement

Dans les samizdats comme dans Rabotnitsa, le fait que l'immense majorité des femmes soviétiques accouchent et/ou avortent reste généralement implicite. L'équation entre féminité et maternité est célébrée dans certains textes dissidents féministes, surtout dans Maria. Seul un texte de Mamonova interroge l'obligation de la maternité :

[Si tu n'es pas mère], tu seras un paria, méprisée par cette société, moins qu'un être humain. [...] Si tu n'es pas mère [...], alors tu seras une « vieille fille » ou une dévergondée. Ne t'agite pas dans les labyrinthes qu'ils ont construits pour toi : ne t'y attendent que des impasses !

La grossesse, c'est ce doux fardeau qu'on désire ! Non seulement l'homme s'en est convaincu lui-même, mais il t'en a aussi convaincu toi. (Collectif 1980a : 44-45).

Si la communauté de destin autour de l'accouchement semble presque inéluctable, pour les femmes, en revanche l'idée qu'il est possible et souhaitable de les aider à échapper à l'avortement revient très souvent, dans les textes dissidents comme dans le magazine féminin. Les solutions envisagées divergent. Une partie des textes du samizdat, se réclamant ou non de la religion, insiste sur les difficultés matérielles qui obligent les femmes enceintes à renoncer à des naissances qu'elles désirent pourtant. Une féministe écrit par exemple : « Je pense que la majorité des femmes qui ont ne serait-ce qu'une fois subi cette torture inhumaine renoncerait à cette opération barbare si elle avait des conditions de vie humaines normales » (ibid. : 57). Cette première voie fait en partie écho à la propagande officielle du régime, qui promet aux femmes de les aider à avoir les nombreux 
enfants qu'elles souhaitent évidemment avoir. Il s'agit en quelque sorte de renvoyer l'État soviétique à ses promesses non tenues.

Une seconde voie consiste à demander un meilleur accès à la contraception, ce qui commençait à devenir pensable sous Brejnev. Cette demande n'apparaît pas dans le premier numéro de $F \& R$, mais elle est évoquée ensuite de façon ponctuelle, aussi bien dans $F \& R$ que dans Maria. Le courant féministe orthodoxe présente l'avortement comme un «péché », mais ne semble pas avoir développé de position unifiée et rigide autour de la question du contrôle des naissances. Un texte, notamment, raconte un travail de relecture de ses avortements passés, et un cheminement qui amène d'un sentiment de «joie » et de « délivrance » après une IVG à de la repentance : « Désormais, le péché de l'infanticide me tourmente et me déchire. Je me repens devant Dieu, et je ne commettrai plus de crimes » (Collectif 1982 : 55-58). Le texte insiste sur le pardon que peuvent obtenir les femmes ayant avorté, vu les conditions de vie en URSS. L'auteure parle de sa " compassion » envers ses « amies désespérées » qui envisagent l'IVG : «Il est déjà difficile de nourrir et d'élever ne serait-ce qu'un enfant », et il faut faire face « l'absence complète de moyens de contraception » : « Dieu, pardonne-nous, aide-nous, protège-nous toutes!» (ibid.). Le texte relate également la recherche désespérée d'un stérilet resté inaccessible, en laissant complètement hors-champ la question du caractère licite ou non de cette méthode contraceptive ${ }^{14}$. Les deux voies pour faire reculer l'avortement - favoriser la maternité ou bien la contraception - ne s'excluent pas mutuellement.

Du côté de Rabotnitsa, la demande de contraception est la seule à être mise en avant. La longue lettre inaugurale sur l'avortement réclame un meilleur accès à la contraception pour faire reculer l'IVG, mais insiste aussi sur le fait que celle-ci n'a pas vocation à disparaître complètement :

Heureusement que l'avortement est autorisé officiellement chez nous. Les gens doivent avoir le droit de décider eux-mêmes la question d'avoir un enfant ou non. Mais ce n'est pas assez d'autoriser cette opération dans les hôpitaux. [...] Il faut aussi se soucier du fait que cette mesure humaniste soit appliquée avec des méthodes vraiment humaines.

Nous allons faire en sorte qu'il y ait moins d'avortements. Mais ceux qui seront tout de même inévitables, dans la mesure du possible, nous les adouciront/simplifieront [oblegchim ${ }^{15}$

\footnotetext{
${ }^{14}$ Le stérilet s'avérera pourtant une méthode controversée, voire condamnée par l'Église orthodoxe russe, considérée comme « abortive » (Luehrmann 2015)

${ }^{15}$ Rabotnitsa, 1986, $\mathrm{n}^{\circ} 10$.
} 
Ce deuxième extrait correspond en fait à un point de convergence important entre des discours en partie divergents sur la question, dans les samizdats et dans Rabotnitsa: même si certaines féministes, notamment orthodoxes, considèrent qu'il ne devrait pas du tout y avoir d'avortements, ce qui est largement partagé, dans les deux corpus, c'est le constat de leur prévalence massive en URSS, et la critique des conditions « inhumaines » dans lesquelles ils se déroulent.

\section{Les souffrances physiques et psychologiques}

Dans les samizdats et dans les courriers publiés dans Rabotnitsa, les souffrances physiques et psychologiques ressenties lors des avortements, d'une part, et lors des accouchements, d'autre part, sont soit abordées séparément, soit rapprochées et mises sur le même plan - ce rapprochement est assez central du côté des féministes, et plus anecdotique du côté du magazine féminin. Le champ lexical de la souffrance est omniprésent, et dans les deux corpus, au sujet des deux actes médicaux, on retrouve des termes comme «torture » ou «tourments». La revendication d'anesthésies (suffisantes) lors des avortements émerge comme une évidence, car il s'agit de la procédure en principe standard, mais souvent bafouée ; il est plus ou moins courant de verser un pot-de-vin pour espérer être (bien) anesthésiée. On notera que l'amélioration que pourrait constituer la substitution du curetage par l'aspiration n'est jamais abordée. Pour l'accouchement, l'anesthésie ne fait pas partie de la procédure standard. Des textes dissidents et des courriers de lectrices en déplorent l'absence. Toutefois, aucune technique précise d'anesthésie n'est jamais demandée. Si dans les années 1980, la péridurale commence à se diffuser à l'Ouest, elle reste inconnue et impensable pour le grand public en URSS. La seule référence précise à une technique d'anesthésie provient des soignant·e's écrivant à Rabotnitsa, qui avouent leur impuissance face au manque de moyens :

Vous demandez : « - Pourquoi vous n’anesthésiez pas ? ». Parce que le Promedol [N.D.A. : un analgésique opioïde] (le produit utilisé en obstétrique) ne supprime par les douleurs de l'accouchement, et on n'a qu'un seul appareil pour toutes les parturientes. Et comment décider qui en a le plus besoin ? $^{16}$

Par ailleurs, pour une partie des auteures de textes dissidents et de courriers au magazine, une large suppression des douleurs de l'accouchement semble tout simplement inimaginable : elles se contentent de se plaindre de ne pas avoir été anesthésiées au moment où elles ont été « recousues ».

${ }^{16}$ Rabotnitsa, 1989, n 2. 
Ce moment des points de suture est souvent mentionné comme particulièrement douloureux, généralement sans plus de précisions sur l'acte. Des déchirures sont parfois évoquées, mais les épisiotomies ne sont jamais précisément nommées : la question de savoir si une partie d'entre elles serait évitable ne peut donc pas être posée. On devine, à travers ces non-dits autour des techniques d'anesthésie et des épisiotomies, à quel point les parturientes étaient privées d'explications sur le déroulement des soins.

Dans les deux corpus, des femmes se plaignent non seulement de souffrir en accouchant, mais aussi de la «honte de crier», intériorisée ou explicitement inculquée par des réprimandes de médecins, qui peuvent dire que c'est «vulgaire » ou bien nocif pour l'enfant à naître. Aucune femme ne semble avoir appris la méthode «psychoprophylactique » pour gérer la douleur, et les sources suggèrent que même sans préparation, on attendait des Soviétiques qu'elles correspondent au modèle de la parturiente silencieuse célébré par Velvovski. Il est frappant que dans les sources étudiées, la question de la préparation à l'accouchement reste pratiquement hors-champ. En outre, il n'est jamais question d'un choix possible entre différentes manières d'accoucher, notamment entre différentes positions, dont certaines pourraient être moins pénibles que d'autres.

Une autre plainte revient ponctuellement, dans les deux corpus : la pénibilité de l'accouchement est jugée aggravée par l'isolement des patientes, par le fait que l'accès aux maternités est strictement interdit à toute personne extérieure. Dans ce type de doléances, la figure du père n'est pas primordiale ; celui-ci est évoqué à peu près autant et au même titre que les grands-parents. Les femmes qui se plaignent semblent appeler de leurs vœux de simples visites de proches, et ne font jamais référence à la possibilité d'être entourée précisément pendant le travail et l'accouchement. Il est frappant de constater que l'inclusion du père dans ce moment, qui devenait relativement incontournable à la même époque en Occident, ne semblait pas pensable en URSS. Les sources étudiées ici vont dans le même sens qu'une enquête réalisée au début des années 1980 aux ÉtatsUnis, auprès d'immigrées soviétiques primo-arrivantes : celles-ci étaient enclines à parler négativement de différents aspects de leurs accouchements vécus en URSS, mais pas de l'absence $\mathrm{du}$ père ; interrogées par le chercheur américain sur cette idée d'inclusion du père, la plupart « semblaient soit amusées soit horrifiées »(Ispa $1983: 10)$.

\section{Le manque de moyens du système de santé}

Un autre enjeu traverse les témoignages et les revendications autour des souffrances physiques et psychologiques des soins gynécologiques, dans les textes féministes et les courriers à la presse : 
l'attitude du personnel soignant est très critiquée, et même un peu plus souvent abordée que le manque d'accès à l'anesthésie. Les mêmes termes reviennent de façon récurrente : le personnel est jugé « indifférent », « cruel », « offensant», " grossier », en gynécologie comme ailleurs. Les femmes sont « traitées comme du bétail » (Collectif 1980a :34), « le malade » est « un prisonnier » (Mamonova $1982:$ 38), la médecine est comme du «travail à la chaîne » dans une « usine ${ }^{17}$. Deux dissidentes féministes, dans $F \& R$, tiennent à analyser les causes structurelles de ces maltraitances. Selon Mamonova :

Les médecins des hôpitaux sont généralement des femmes, et ils peuvent avoir jusqu'à 30 patients par jour, ce qui élimine toute approche créative dans leur travail, ainsi que l'individualité des patients. [...] Les raisons derrière l'attitude du personnel sont évidentes [...]. Les salaires sont bas, le personnel en sous-effectif [...]. (Mamonova 1984 : xix)

Selon Kari Ounskova, «il ne faut pas accuser les infirmières » qui doivent travailler dans «l'horrible entassement des salles d'hôpital », dans l'odeur des excréments : «cela sème dans [leur] âme [...] l'ivraie vivace de la cruauté » (Mamonova 1982:38). Toutes les deux rattachent les maltraitances gynécologiques au sous-financement du système de santé. Dans Rabotnitsa, les femmes qui témoignent de leur expérience de patiente ne sont pas dans cette démarche de disculpation, mais elles critiquent très souvent le manque de moyens des établissements de santé. En outre, la lettre écrite par une sage-femme au nom de l'ensemble de son service met l'attitude du personnel sur le compte de ses conditions de travail. Elle raconte que lorsqu'une patiente lui dit «j'ai mal, restez un peu avec moi »: «je resterais avec plaisir, parler un peu avec elle, mais je cours faire autre chose $»^{18}$.

Plusieurs lettres de patientes se plaignent en fait d'un personnel tellement débordé que cela pose des problèmes encore plus graves que ceux des douleurs et du manque de respect: des soins arrivent trop tard, des naissances ont lieu sans assistance dans les couloirs des hôpitaux, et comme le souligne la rédaction, la mortalité infantile est bien plus élevée qu'à l'Ouest, de nombreuses morts de nouveaux nés sont évitables. Les deux corpus relient la question des souffrances en gynécologie avec celle du dénuement du système de santé, s'agissant du manque d'anesthésie, des conditions de travail du personnel, mais aussi plus largement du manque d'équipements, et des problèmes d'hygiène. Une lectrice demande : « Pourquoi nous n'avons pas de linge à usage unique,

\footnotetext{
${ }^{17}$ Rabotnitsa, 1986, n¹0.

${ }^{18}$ Rabotnitsa, 1989, n²2.
} 
qui pourrait nous débarrasser pour toujours du staphylocoque ? ${ }^{19}$. D'autres lettres dénoncent les problèmes de chauffage, les lavabos cassés, les cafards, le manque d'antiseptique ou de stéthoscopes.

Pour finir, plusieurs femmes, aussi bien parmi les dissidentes que parmi les lectrices de Rabotnitsa, poussent leur critique plus loin, en posant la question des choix qui sont faits dans l'allocation des ressources. Pour Mamonova, l'absence d'anesthésie lors des accouchements s'explique aussi par une certaine vision des douleurs féminines en obstétrique : «À la question “Comment vous pouvez tolérer ça ?!", les médecins répondent : "Notre objectif, c'est les enfants.

La femme pourra tout supporter..." "(Collectif 1980a : 48). Une lectrice de Rabotnitsa soulève aussi la question des inégalités de genre dans la prise en charge des douleurs : «Pourquoi à un homme en bonne santé, on lui arrache une dent en lui faisant une piqûre, tandis qu'à une femme, pardon, on la recoud sans la moindre anesthésie ? Pourquoi nous on a ces terribles tourments des accouchements et des avortements ? $»^{20}$. Un lecteur se demande : «Est-ce que la brutalité des avortements [sans anesthésie], ce ne serait pas aussi pour que ça ne leur prenne pas de recommencer? $»^{21}$. Les souffrances en gynécologies peuvent alors être dénoncées comme le résultat de choix misogynes, à l'intérieur des contraintes du budget de la santé, mais aussi plus largement du budget de l'État. Selon Mamonova, «l'homme» se moque des souffrances de l'accouchement, " il préfère organiser une nouvelle guerre ", "il préfère inventer une nouvelle fusée » (Collectif 1980a : 48). Une lectrice de la presse féminine ironise de façon similaire sur les priorités de l'économie planifiée : «On envoie des fusées dans l'espace, mais on est incapable de créer des moyens de contraception fiables... $»^{22}$.

\section{Conclusion}

Les critiques des maltraitances gynécologiques qui ont émergé d'abord dans la dissidence féministe, vers 1980, puis dans les courriers de lectrices de la perestroïka, ont largement convergé. Pourtant, il n'y a pas pu y avoir « d'appropriation ordinaire des idées féministes » (Jacquemart et Albenga 2015), en contexte de répression et de censure. On peut faire l'hypothèse qu'à la fin de

${ }^{19}$ Rabotnitsa, $1989, \mathrm{n}^{\circ} 2$.

${ }^{20}$ Rabotnitsa, $1987, \mathrm{n}^{\circ} 7$.

${ }^{21}$ Rabotnitsa, $1986, \mathrm{n}^{\circ} 10$.

${ }^{22}$ Rabotnitsa, $1989, \mathrm{n}^{\circ} 2$. 
l'ère Brejnev, une «critique ordinaire des patientes" (Cantelli 2015) s'exprimait dans des conversations féminines au sujet des souffrances gynécologiques, qui constituaient une pratique routinisée. Cette pratique infrapolitique a pu servir de matrice commune à des publications féministes confidentielles, puis à un abondant courrier de plaintes à la presse féminine grand public. Les critiques ordinaires et féministes ont convergé autour de plusieurs revendications : des méthodes contraceptives permettant d'éviter les IVG à répétition; des anesthésies lors des avortements et des accouchements ; un personnel soignant plus disponible et plus respectueux, dispensant des soins plus individualisés ; l'autorisation des visites des proches dans les maternités. En revanche, par contraste avec les mobilisations féministes et de patientes en Occident, différents enjeux sont restés impensables et indicibles, en raison de la censure et de l'isolement de l'URSS par rapport au reste du monde: la péridurale, la participation du père à l'accouchement, l'accouchement à domicile, le choix de la position d'accouchement, la pertinence du recours à l'épisiotomie, ou encore la méthode d'avortement par aspiration.

Cependant, cette spécificité ne doit pas nous empêcher de nous poser la question de l'inclusion du cas soviétique dans une histoire véritablement globale des mobilisations féminines et féministes autour de la gynécologie, qui reste largement à écrire. Le présent article fournit quelques pistes. On peut noter, notamment, que contrairement aux féministes états-uniennes des années 1970, les dissidentes féministes soviétiques n'ont pas concentré leurs critiques sur la figure du gynécologue homme tout puissant, dans un contexte où leurs médecins étaient généralement des femmes, fonctionnaires et sous-payées. Les analyses d'Ilana Löwy (2005 : 103) semblent bien pouvoir s'appliquer au cas soviétique : selon elle, contrairement à ce qui se passait aux États-Unis, « dans des pays dotés d'un système de santé étatique », comme la France ou la Grande-Bretagne, « la cible d'action collective [des féministes] [était] souvent le gouvernement, plutôt que les médecins euxmêmes ». En URSS, la critique féministe de la gynécologie, comme celle des lectrices de Rabotnitsa, était aussi une critique du sous-financement du système de santé, et de la priorité accordée par l'État soviétique au budget de la conquête spatiale et de la Guerre froide.

Mona Claro (Université de Liège) 


\section{Références}

Achin, C., \& Naudier, D. (2009) « La libération par Tupperware ?. Diffusion des idées et pratiques féministes dans de nouveaux espaces de sociabilité féminine », Clio. Femmes, Genre, Histoire, (29) : 131-140.

Avdeev, A., \& Troitskaia, I. (1991) "Contraception and Abortion in the USSR: Experience of 1980's ", communication présentée à la European Population Conference, Paris, 21-25 octobre.

Blum, A. (2018) « Politiques du secret et inefficacité politique », Revue d'études comparatives EstOuest, 1(1) : 127-173.

Bridger, S. (1990) «Women in contemporary Soviet society », Economy and Society, 19(4) : 511 530.

Cantelli, F. (2015) " "Inacceptable !" : critiquer et faire valoir ses droits en tant que patient », Sciences sociales et santé, 33(2) : 107-115.

Charpenel, M. (2016) «Les groupes de parole ou la triple concrétisation de l'utopie féministe », Education et sociétés, 37(1) : 15-31.

Claro, M. (2018) Ni hasard ni projet. Genre, sexualité et procréation pendant la jeunesse en Russie (années 1970 - années 2010), Thèse de doctorat en sociologie. Paris : EHESS.

Collectif (1980a) Zhenshchina i Rossiia. Paris : des Femmes.

Collectif (1980b) Rossiianka. Paris : des Femmes.

Collectif (1982), Maria. Frankfort sur le Main.

Davis, C. (1998) « Morbidité, mortalité et réformes du système de santé dans les États en transition de l'ex-U.R.S.S. et de l'Europe de l'Est », Revue d'études comparatives Est-Ouest, 29(3) : 133185.

Fitzpatrick, S. (1996) «Supplicants and Citizens: Public Letter-Writing in Soviet Russia in the 1930s », Slavic Review, 55(1) : 78-105.

Fürst, J. (2006) « In Search of Soviet Salvation: Young People Write to the Stalinist Authorities », Contemporary European History, 15(3) : 327-345.

Harting, K. (2013) Transferts franco-russes? L'almanach Femmes et Russie comme point à l'intersection des féminismes dans les années 1970-1980, Mémoire de master en histoire. Paris : Université Paris 7.

Hrešanová, E., \& Michaels, P. A. (2018) «Socialist Science Across Borders », Revue d'études comparatives Est-Ouest, $\mathrm{n}^{\circ} 1(1): 45-69$.

Ispa, J. (1983) « Soviet and American Childbearing Experiences and Attitudes: A Comparison », Slavic Review, 42(1) : 1-13.

Jacquemart, A., \& Albenga, V. (2015) «Pour une approche microsociologique des idées politiques », Politix 109(1) : 7-20.

John Snow Inc. (2012) Transforming Health Care for Mothers and Babies in the Former Soviet Union.

Knibiehler, Y. (2010) «Une migration décisive », Spirale, 54(2) : 39-45.

Löwy, I. (2005) «Le féminisme a-t-il changé la recherche biomédicale ?», Travail, genre et sociétés, 14(2) : 89-108. 
Luehrmann, S. (2015) « Abortive or Non-Abortive? Russian Orthodox Positions on Birth Control as Strategic Ambiguity 》, communication présentée à la 4th Health in Transition Conference: Challenges of Health, Demographic Changes, and Wellbeing in Post-socialist Societies. Riga : University of Latvia, 26 août.

Mamonova T. (1984), Women and Russia. Boston : Beacon Press.

Mamonova T. (1982) Voix de femmes en Russie, Paris : Denoël-Gonthier.

Michaels, P. A. (2014) Lamaze: An International History. Oxford : OUP USA.

Morgen, S. (2002) Into Our Own Hands: The Women's Health Movement in the United States, 1969-1990. New Brunswick : Rutgers University Press.

Nérard, F.-X. (1999) « Entre plainte et délation : les « signaux » en U.R.S.S. (1928-1939) », Revue d'études comparatives Est-Ouest, 30(1) : 5-30.

Pavard, B. (2009) «Contraception et avortement dans Marie-Claire (1955-1975) : de la méthode des températures à la méthode Karman », Le Temps des médias (12) : 100-113.

Pavard, B. (2017) «Marie Claire, magazine féministe?", Vingtième Siècle. Revue d'histoire, $136(4): 131-146$.

Quéré, L. (2019) «Les formes ordinaires du consentement. Consciences du droit dans la consultation gynécologique », Droit et société, 102(2) : 413-432.

Riska, E. (2001) Medical Careers and Feminist Agendas: American, Scandinavian, and Russian Women Physicians. New York : Aldine de Gruyter.

Rivkin-Fish, M. (2005) Women's Health in Post-Soviet Russia: The Politics of Intervention. Indianapolis : University of Indiana Press.

Ruault, L. (2017) Le spéculum, la canule et le miroir. Les MLAC et mobilisations de santé des femmes, entre appropriation féministe et propriété médicale de l'avortement (France, 19721984), Thèse de doctorat en science politique. Lille : Université Lille 2.

Ruthchild, R. (2017) « Feminist Dissidents in the "Motherland of Women's Liberation": Shattering Soviet Myths and Memory », in B. Molony, Women's Activism and "Second Wave" Feminism, pp. 99-123. Londres : Bloomsbury Academic. ss.

Sidorevich, A. (2017) Un féminisme dissident orthodoxe : une étude historique de la revue Maria en Union Soviétique (1979-1982), Mémoire de master 1 en histoire. Moscou : Collège universitaire français de Moscou.

Talaver, A. (2017) Samizdat Magazines Of The Soviet Dissident Women's Groups, 1979-1982: A Critical Analysis, Mémoire de master en gender studies. Budapest: Central European University.

Tchouikina, S. (1996) "Uchastie zhenshchin v dissidentskom dvizhenii (1956-1986)», in E. Zdravomyslova \& A. Temkina, Gendernoe Izmerenie Sotsial'noi i Politicheskoi Aktivnosti v Perekhodnyi Period, pp. 61-81. Saint-Pétersbourg : TsNSI.

Vuille, M. (2016) «Le respect dans les soins gynécologiques: un enjeu de mobilisation féministe », Chronique féministe (118) : 5-7.

Vuille, M. (2017) «L'obstétrique sous influence : émergence de l'accouchement sans douleur en France et en Suisse dans les années 1950 », Revue d'histoire moderne contemporaine, 64-1(1) : 116-149. 